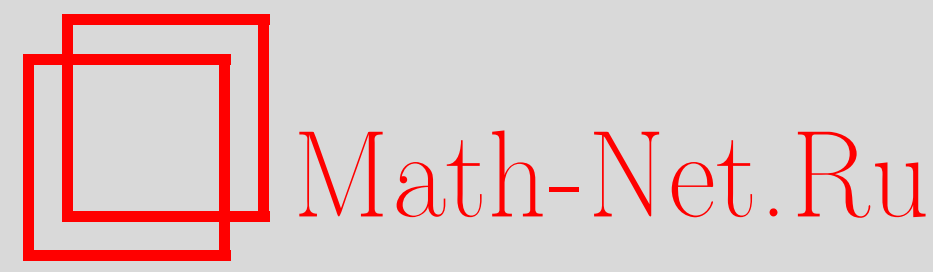

M. В. Кочетов, Тождества smash-произведения универсальной обертывающей супералгебры Ли и групповой алгебры, Матем. сб., 2003, том 194, номер 1, 87-102

DOI: https://doi.org/10.4213/sm707

Использование Общероссийского математического портала Math-Net.Ru подразумевает, что вы прочитали и согласны с пользовательским соглашением http://www . mathnet.ru/rus/agreement

Параметры загрузки:

IP : 52.205 .19 .152

26 апреля 2023 г., $17: 04: 54$ 
УДК 512.552 .4

\author{
М.В. Кочетов
}

\title{
Тождества smash-произведения универсальной обертывающей супералгебры Ли и групповой алгебры
}

Пусть $H$ - алгебра Хопфа, $A-H$-модульная алгебра. Тогда мы можем образовать smash-произведение $A \# H$, которое является обобщением обычного тензорного произведения (последнее возникает, если действие $H$ на $A$ тривиально). В статье рассматривается вопрос, когда $A \# H$ удовлетворяет полиномиальному тождеству. Вводятся дельта-множества подходящего вида и даются необходимые условия на действие $H$ на $A$ в терминах этих дельта-множеств для определенного класса алгебр. Основная теорема посвящена частному случаю, когда $H$ является групповой алгеброй, которая действует на супералгебре Ли $L$ характеристики нуль. В этом случае полученные результаты о дельта-множествах, совместно с известными фактами о групповых и универсальных обертьвающих алгебрах, позволяют дать необходимые и достаточные условия наличия полиномиального тождества в $U(L) \# H$.

Библиографияя: 11 названий.

\section{$\S$ 1. Введение}

Пусть $\mathbb{k}$ - некоторое поле. Все рассматриваемые нами векторные пространства, алгебры, тензорные произведения и т. п. будут над полем $\mathbb{k}$. Пусть $H$ - алгебра Хопфа. Мы будем использовать так называемые “сигма-обозначения" для коумножения $H$ :

$$
\Delta: H \rightarrow H \otimes H: h \mapsto \sum h_{(1)} \otimes h_{(2)} .
$$

Эта запись является символической в том смысле, что $h_{(1)}$ и $h_{(2)}$ не являются какими-то определенньми элементами $H$. Коединица $H$ будет обозначаться

$$
\varepsilon: H \rightarrow \mathbb{k}: h \mapsto \varepsilon(h) .
$$

Напомним определение объекта, который, за неимением лучшего термина, мы будем называть "smash-произведением" (от английского "smash product", cм. $[1 ;$ п. 4.1]).

ОПРЕДЕЛЕНИЕ 1.1. Пусть $H$ - алгебра Хопфа, а $A$ - ассоциативная алгебра с единицей. Пусть задано действие $H$ на $A$ :

$$
H \otimes A \rightarrow A: h \otimes a \mapsto h \cdot a,
$$

так что $A$ преврашается в $H$-модульную алгебру, т.е. $A$ является $H$-модулем и, кроме того, для любых $h \in H, a, b \in A$ выполняется

$$
\begin{gathered}
h \cdot 1=\varepsilon(h) 1, \\
h \cdot(a b)=\sum\left(h_{(1)} \cdot a\right)\left(h_{(2)} \cdot b\right) .
\end{gathered}
$$

(C) М. В. Кочетов 2003 
Тогда smash-произведение $A \# H$ определяется как векторное пространство $A \otimes H$, снабженное умножением

$$
(a \# h)(b \# k)=\sum a\left(h_{(1)} \cdot b\right) \# h_{(2)} k,
$$

где $h, k \in H, a, b \in A$ и мы пишем $a \# h$ вместо $a \otimes h$.

Легко видеть, что $A \# H$ является ассоциативной алгеброй с единицей $1 \# 1$, причем $A$ и $H$ изоморфны подалгебрам $A \# 1$ и $1 \# H$ соответственно. Если действие $H$ на $A$ тривиально, получаем обычное тензорное произведение.

Smash-произведение возникает, в частности, в структурной теории алгебр Хопфа. Например, классическая теорема Костанта, Картье и Габриеля (см. [1; п. 5.6]) утверждает, что всякая точечная кокоммутативная алгебра Хопфа изоморфна smash-произведению связной алгебры Хопфа и групповой алгебры некоторой групшы. Причем в случае поля характеристики нуль структура связной компоненты тоже известна - это универсальная обертывающая некоторой алгебры Ли.

Представляет интерес вопрос, когда $A \# H$ удовлетворяет полиномиальному тождеству? Если ограничиться случаем $H=\mathbb{k} G$ - групповая алгебра некоторой группы $G$, то алгебра $A \# \mathbb{k} G$ известна также в литературе под названием косого группового кольца группы $G$ с коэффициентами в $A$.

Критерий того, когда такое групповое кольцо удовлетворяет полиномиальному тождеству в предположении, что $A$ является $G$-полупервичным кольцом, дан в [2; гл. 23] (там рассматривается даже несколько более общий случай: умножение элементов из $G$ "подкручено" с помощью коцикла). Надо отметить, что этот критерий не дает явного описания структуры $A$ или действия $G$ на $A$.

Другой подход к проблеме - ограничиться рассмотрением $A$ какого-нибудь конкретного типа. Например, критерий наличия тождества в $A \# \mathbb{k} G$ в случае $A=$ $U(L)$ или $u(L)$ - (ограниченная) обертываюшая некоторой (ограниченной) алгебры Ли $L$, на которой $G$ действует автоморфизмами, дан в [3]. В этом случае удается достичь конкретного описания структуры $G, L$ и действия $G$ на $L$, не предполагая при этом $G$-полупервичности. В настоящей статье мы получим ответ для $A=U(L)$, где $L=L_{0} \oplus L_{1}$ - некоторая супералгебра Ли характеристики нуль. Но прежде приведем здесь критерии наличия тождества в $U(L)$ и $\mathbb{k} G$, взятых в отдельности (см. [4] и [5]: нам потребуется лишь случай характеристики нуль, положительная характеристика рассматривается в [6] и [7]).

Теорема 1.2. Пусть $L=L_{0} \oplus L_{1}-$ супералгебра Ли над полем $\mathbb{k}$ характеристики нуль. Универсальная обертьввающая алгебра $U(L)$ удовлетворяет полиномиальному тождеству тогда и только тогда, когда $L_{0}$ абелева и существует такой $L_{0}$-подмодуль $M \subset L_{1}$, что $[M, M]=0$, а $L_{1} / M u\left[L_{0}, M\right]$ конечномерны.

Теорема 1.3. Пусть $G$ - группа, $\mathbb{k}$ - поле. Групповая алгебра $\mathbb{k} G$ удовлетворяет полиномиальному тождеству тогда и только тогда, когда существует подгруппа $A \subset G$ конечного индекса, абелева в случае char $\mathbb{k}=0$ и $p$-абелева в случае char $\mathbb{k}=p>0$. 
Нам также потребуется критерий для smash-произведения универсальной обертывающей обычной алгебры Ли характеристики нуль и групповой алгебры (см. [8], вытекает также из результатов в [9]).

ТЕОРема 1.4. Пусть $L$ - алгебра Ли над полем $\mathbb{k}$ характеристики нуль. Пусть группа $G$ действует на $L$ автоморфизмами. Тогда $U(L) \# \mathbb{k} G$ удовлетворяет полиномиальному тождеству тогда и только тогда, когда L абелева и найдется абелева подгруппа $A \subset G$ конечного индекса, действующая тривиально на L.

Основным результатом настоящей статьи является следующий критерий наличия полиномиального тождества в smash-произведении универсальной обертывающей супералгебры Ли и групповой алгебры надполем характеристики нуль. Под автоморфизмом градуированной алгебры мы понимаем автоморфизм, оставляющий компоненты градуировки инвариантньми.

Теорема 1.5. Пусть $L=L_{0} \oplus L_{1}-$ супералгебра Ли над полем $\mathbb{k}$ характеристики нуль. Пусть группа $G$ действует на $L$ автоморфизмами градуированной алгебры. Тогда sтаsh-произведение $U(L) \# \mathbb{k} G$ удовлетворяет полиномиальному тождеству тогда и только тогда, когда существуют такие $L_{0}$ - и G-инвариантные подпространства $N \subset M \subset L_{1}$, а также подгруппа $A \subset G$, что выполняются следующие условия:

1) $N$ и $L_{1} / M$ конечномерны;

2) $\left[L_{0}, L_{0}\right]=[M, M]=0 u\left[L_{0}, M\right] \subset N$;

3) А абелева и имеет конечный индекс в $G$;

4) А действует тривиально на $L_{0}$ и на $M / N$.

Достаточность этих условий доказьвается сравнительно просто. Необходимость 1) и 2) вытекает из теоремы 1.2, а 3 ) - из теоремы 1.3. Единственная трудность здесь - показать, что подпространства $N$ и $M$ могут быть выбраны $G$-инвариантными. Мы займемся этим в $\S 3$. Для доказательства необходимости условия 4) нам потребуется теория дельта-множеств для действия групп, развиваемая в $\S 2$ и представляюшая самостоятельньй интерес. Следует заметить, что идея дельта-множеств этого типа была предложена в [3], где она применяется для изучения smash-произведения ограниченной обертывающей ограниченной алгебры Ли и групповой алгебры.

Проиллюстрируем на примерах, что теорема 1.5 не может быть усилена путем отбрасывания $N$, т.е. нельзя утверждать, что найдется такое подпространство $M$ конечной коразмерности, что $L_{0}$ и $A$ действуют тривиально на $M$.

ПримеР 1.6. Возьмем $L_{0}=\left\langle x_{1}, x_{2}, \ldots\right\rangle, L_{1}=\left\langle z, y_{1}, y_{2}, \ldots\right\rangle$ и $G=\{1\}$, где $\left[L_{0}, L_{0}\right]=\left[L_{1}, L_{1}\right]=0$ и

$$
\left[x_{i}, z\right]=0, \quad\left[x_{i}, y_{j}\right]=\delta_{i j} z, \quad i, j=1,2, \ldots
$$

Тогда $U(L)$ удовлетворяет тождеству $[X, Y]^{2}=0$ и условиям теоремы с $M=L_{1}$, $N=\langle z\rangle$, однако, как легко видеть, централизатор $L_{0}$ есть одномерное пространство $\langle z\rangle$, имеюшее в $L_{1}$ бесконечную коразмерность. 
ПримеР 1.7. Положим $L_{0}=0, L_{1}=\left\langle z, y_{1}, y_{2}, \ldots\right\rangle$ и $G=\mathbb{Z}^{\mathbb{N}}=\left\langle g_{1}, g_{2}, \ldots\right\rangle$, причем

$$
g_{i} \cdot z=z, \quad g_{i} \cdot y_{j}=y_{j}+\delta_{i j} z, \quad i, j=1,2, \ldots .
$$

Тогда $U(L) \# \mathbb{k} G$ удовлетворяет тождеству $[X, Y]^{2}=0$ и условиям теоремы с $M=L_{1}, N=\langle z\rangle$ и $A=G$, однако пространство неподвижных точек $G$ есть $\langle z\rangle$, имеющее бесконечную коразмерность, причем ситуация не изменится, если мы заменим $G$ какой-либо подгруппой конечного индекса.

\section{§2. Дельта-множества}

В этом параграфе мы несколько обобщаем результаты [3] о дельта-множествах для действия группы, в частности, чтобы они были применимы в нашей ситуации. Характеристика поля в большинстве результатов произвольна.

Начнем с общего наблюдения, принадлежащего Ю. А. Бахтурину. Пусть $H-$ алгебра Хопфа, $A-H$-модульная алгебра. Если $A \# H$ удовлетворяет полиномиальному тождеству, то имеется "слабое" тождество следуюшего вида, несущее информацию о структуре действия $H$ на $A$ (доказательство в случае $H=\mathbb{k} G$ см. в [3], общий случай - в [10]).

ПрЕДЛОЖенИЕ 2.1. Если smash-произведение A\#Н является PI, то найдутся такие натуральное число $n$ и коэффициенты $\gamma_{\pi} \in \mathbb{k}, \pi \in S_{n}$, не все равнье нулю, что

$$
\sum_{\pi \in S_{n}} \gamma_{\pi}\left(h_{1} \cdot X_{\pi(1)}\right) \cdots\left(h_{n} \cdot X_{\pi(n)}\right)=0
$$

для любих $h_{1}, \ldots, h_{n} \in H, X_{1}, \ldots, X_{n} \in A$.

Допустим теперь, что в $A$ имеется $H$-подмодуль $V$, порождающий $A$ как алгебру с единицей. Мы можем ввести два типа дельта-множеств для действия $H$ на $V$ (которое определяет действие на всей $A$ ):

$$
\begin{aligned}
& \delta_{H}^{m}(V)=\{v \in V \mid \operatorname{dim}(H \cdot v) \leqslant m\}, \quad \delta_{H}(V)=\bigcup_{m} \delta_{H}^{m}(V), \\
& \delta_{V}^{m}(H)=\{h \in H \mid \operatorname{dim}(h \cdot V) \leqslant m\}, \quad \delta_{V}(H)=\bigcup_{m} \delta_{V}^{m}(H),
\end{aligned}
$$

где $m=0,1, \ldots$.

Непосредственно проверяются следующие свойства.

Лемма 2.2. 1) Если $x \in \delta_{H}^{i}(V), y \in \delta_{H}^{j}(V)$, mо $\alpha x+\beta y \in \delta_{H}^{i+j}(V)$, где $\alpha, \beta \in \mathbb{k}$.

2) Все $\delta_{H}^{m}(V)$ являются $H$-инвариантными множсествами.

3) Если $\Phi: V \rightarrow V$ - гомоморфизм $H$-модулей, то $\Phi\left(\delta_{H}^{m}(V)\right) \subset \delta_{H}^{m}(V)$.

4) $E c л и \quad h \in \delta_{V}^{i}(H), k \in \delta_{V}^{j}(H)$, mо $\alpha h+\beta k \in \delta_{V}^{i+j}(H)$, где $\alpha, \beta \in \mathbb{k}$.

5) Все $\delta_{V}^{m}(H)$ являются инвариантными относительно правых и левых умножений на әлементы $H$.

Из леммы вытекает, что $\delta_{H}(V)-H$-подмодуль $V$, а $\delta_{V}(H)$ - двусторонний идеал $H$ (но, вообще говоря, не идеал Хопфа!). Поскольку $\delta_{H}^{m}(V)$ и $\delta_{V}^{m}(H)$ не обязаны быть подпространствами, нам потребуется понятие коразмерности подмножества векторного пространства. 
ОПРЕДЕЛЕНИЕ 2.3. Пусть $W$-векторное пространство, $T \subset W$-подмножество. Мы будем писать $\operatorname{dim} W / T=n$, если найдется такое подпространство $U \subset W$ размерности $n$, что $W=U+T$ и $n$ - минимальное натуральное число с этим свойством.

Используя порождающее множество $V$, мы можем определить возрастающую фильтрацию на алгебре $A$

$$
A=\bigcup_{m \geqslant 0} A_{m}, \quad A_{m}=V^{m}
$$

а также ассоциированную градуированную алгебру

$$
\operatorname{gr} A=\bigoplus_{m \geqslant 0} \operatorname{gr}_{m} A, \quad \operatorname{gr}_{m} A=A_{m} / A_{m-1}, \quad m \geqslant 1, \quad \operatorname{gr}_{0} A=\langle 1\rangle .
$$

Поскольку $V \subset A-H$-подмодуль, gr $A$ естественным образом преврашается в $H$-модульную алгебру. В дальнейшем мы будем предполагать, что $V$ не содержит $1 \in A$, так что его можно естественньм образом отождествить с $\operatorname{gr}_{1} A$. Отсюда получаем, что вся информация о действии $H$ на $A$ на самом деле содержится в действии $H$ на gr $A$.

Мы будем использовать следующие обозначения. Пусть $V$ - векторное пространство над $\mathbb{k}$, тогда $S(V)$ - его симметрическая алгебра, а $\Lambda(V)$ - внешняя алгебра. Если char $\mathbb{k}=p>0$, то через $V^{[p]}$ мы будем обозначать подпространство алгебры $S(V)$, порожденное элементами $v^{p}, v \in V$.

ПреДЛОЖенИЕ 2.4. Пусть $A-H$-модульная алгебра. Пусть $H$-подмодуль $V \subset A$ не содержит 1 и порождает $A$ как алгебру с единищей. Предположим далее, что определяемая $V$ градуированная алгебра gr $A$ изоморфна $S(V), \Lambda(V)$ или $S(V) / \operatorname{ideal}\left(V^{[p]}\right)$ (в случае $\operatorname{char} \mathbb{k}=p>0$ ). Тогда из виьполнения "слабого" тождества (1) степени $n$ для любых $h_{1}, \ldots, h_{n} \in H$, $X_{1}, \ldots, X_{n} \in V$ вытекает

1) $\operatorname{dim} V / \delta_{H_{2}}^{n^{2}}(V)<n$,

2) $\operatorname{dim} H / \delta_{V}^{n^{2}}(H)<n$.

ДоказАТЕЛЬСтво. Поскольку $V$ вкладывается в $\operatorname{gr} A$ как $H$-модуль и тождество (1) выполняется для действия $H$ на gr $A$, мы можем заменить $A$ на gr $A$. Мы можем также считать без ограничения общности, что в (1) $\gamma_{1} \neq 0$.

1) Следуя идее доказательства теоремы 6.2 в [3], зафиксируем произвольные элементы $v_{1}, \ldots, v_{n} \in V$. Обозначая левую часть (1) за $F$, получаем

$$
F\left(v_{1}, \ldots, v_{n} ; h_{1}, \ldots, h_{n}\right)=\sum_{\pi \in S_{n}} \gamma_{\pi}\left(h_{1} \cdot v_{\pi(1)}\right) \cdots\left(h_{n} \cdot v_{\pi(n)}\right)=0
$$

для любых $h_{1}, \ldots, h_{n} \in H$.

Докажем индукцией по $m$ следующее утверждение: если $v_{1}, \ldots, v_{m} \in V$ удовлетворяют условию

$$
F\left(v_{1}, \ldots, v_{m} ; h_{1}, \ldots, h_{m}\right)=\sum_{\pi \in S_{m}} \gamma_{\pi}\left(h_{1} \cdot v_{\pi(1)}\right) \cdots\left(h_{m} \cdot v_{\pi(m)}\right)=0
$$


для всех $h_{1}, \ldots, h_{m} \in H$, где $\gamma_{1} \neq 0$, то $v_{1}, \ldots, v_{m}$ линейно зависимы по модулю $\delta_{H}^{m^{2}}(V)$. При $m=n$ это как раз дает желаемое.

Итак, если $m=1$, получаем $h_{1} \cdot v_{1}=0$ для всех $h_{1} \in H$ и, в частности, для $h_{1}=1$, так что $v_{1}=0$. Пусть теперь $m>1$. Мы можем разложить левую часть $(2)$ следующим образом:

$$
F\left(v_{1}, \ldots, v_{m} ; h_{1}, \ldots, h_{m}\right)=\sum_{i=1}^{m}\left(h_{1} \cdot v_{i}\right) F_{i}\left(v_{1}, \ldots, \widehat{v}_{i}, \ldots, v_{m} ; h_{2}, \ldots, h_{m}\right)
$$

где $F_{i}$ имеет тот же вид, что и $F$, но степень $m-1$.

Если теперь $F_{1}\left(v_{2}, \ldots, v_{m} ; h_{2}, \ldots, h_{m}\right)=0$ для всех $h_{2}, \ldots, h_{m} \in H$, то по предположению индукции $v_{2}, \ldots, v_{m}$ линейно зависимы по модулю $\delta_{H}^{(m-1)^{2}}(V)$ и доказательство закончено. В противном случае зафиксируем такие $h_{2}, \ldots, h_{m} \in H$, что $F_{1}\left(v_{2}, \ldots, v_{m} ; h_{2}, \ldots, h_{m}\right) \neq 0$. Тогда $f_{i}=F_{i}\left(v_{1}, \ldots, \widehat{v}_{i}, \ldots, v_{m} ; h_{2}, \ldots, h_{m}\right)$, $i=1, \ldots, m,-$ фиксированные элементы $A$, причем $f_{1} \neq 0$. Из (2) и (3) тогда получаем следующее тождество:

$$
\sum_{i=1}^{m}\left(h \cdot v_{i}\right) f_{i}=0 \quad \forall h \in H .
$$

Положим $W=\left\langle h_{i} \cdot v_{j} \mid i=2, \ldots, m, j=1, \ldots, m\right\rangle$, тогда $W$ - подпространство $V$ размерности менњше $m^{2}$. Выберем в $W$ упорядоченный базис и расширим его до базиса $V$. Согласно нашему предположению о структуре алгебры $A$ упорядоченные мономы от элементов базиса $V$ со степенями переменных меньше $p$ в случае $S(V) / \operatorname{ideal}\left(V^{[p]}\right)$ и меньше 2 в случае $\Lambda(V)$ образуют базис $A$. Для элемента $z \in A$ через supp $z$ обозначим множество всех элементов базиса $V$, встречающихся в представлении $z$. Тогда по построению $\operatorname{supp} f_{i} \subset W$ для всех $i=1, \ldots, m$.

Будем теперь уменшшать шаг за шагом количество слагаемых в (4).

Если $H \cdot v_{m} \subset W$, то $v_{m} \in \delta_{H}^{m^{2}}(V)$ и доказательство закончено. Иначе выберем такой $d \in H$, что $e=d \cdot v_{m} \notin W$. Без ограничения общности мы можем считать, что $e$ включен в наш базис для $V$. Положим $d \cdot v_{j}=\alpha_{j} e+u_{j}, j=1, \ldots, m-1$, где $\operatorname{supp} u_{j}$ не содержит $e$. Подставляя $h=d$ в (4), получаем

$$
e\left(\alpha_{1} f_{1}+\cdots+\alpha_{m-1} f_{m-1}+f_{m}\right)+u_{1} f_{1}+\cdots+u_{m-1} f_{m-1}=0 .
$$

Поскольку $\operatorname{supp} u_{j} f_{j}, j=1, \ldots, m-1$, не содержат элемента $e$, это равенство возможно лишь в случае $e\left(\alpha_{1} f_{1}+\cdots+\alpha_{m-1} f_{m-1}+f_{m}\right)=0$, откуда по тем же соображениям $\alpha_{1} f_{1}+\cdots+\alpha_{m-1} f_{m-1}+f_{m}=0$. Выражая отсюда $f_{m}$ и подставляя обратно в (4), приходим к тождеству

$$
\sum_{i=1}^{m-1}\left(h \cdot\left(v_{i}-\alpha_{i} v_{m}\right)\right) f_{i}=0 \quad \forall h \in H
$$

которое имеет тот же вид, что и (4), но слагаемых на одно меньше. Заметим также, что если нам удастся доказать, что

$$
\widetilde{v}_{1}=v_{1}-\alpha_{1} v_{m}, \ldots, \widetilde{v}_{m-1}=v_{m-1}-\alpha_{m-1} v_{m}
$$


линейно зависимы по модулю $\delta_{H}^{m^{2}}(V)$, то и $v_{1}, \ldots, v_{m}$ будут линейно зависимы по модулю $\delta_{H}^{m^{2}}(V)$.

Повторяя вьшеописанную процедуру $m-1$ раз, мы приходим к соотношению

$$
\left(h \cdot \widetilde{\widetilde{v}}_{1}\right) f_{1}=0 \quad \forall h \in H .
$$

Если теперь $H \cdot \widetilde{\widetilde{v}}_{1} \not \subset W$, то мы можем так выбрать $d \in H$, что $d \cdot{\underset{\widetilde{v}}{1}}_{1}=e \notin W$, и тогда $e f_{1}=0$ влечет $f_{1}=0$-противоречие. Следовательно, $H \cdot \widetilde{\widetilde{v}}_{1} \subset W$, а это означает $\widetilde{\widetilde{v}}_{1} \in \delta_{H}^{m^{2}}(V)$. Доказательство первого утверждения закончено.

2) Доказательство второго утверждения теоремы вполне аналогично доказательству первого (и следует идее [3; теорема 6.3]). Здесь мы вместо (1) используем его эквивалентную форму:

$$
F^{\prime}\left(X_{1}, \ldots, X_{n} ; h_{1}, \ldots, h_{n}\right)=\sum_{\pi \in S_{n}} \gamma_{\pi}^{\prime}\left(h_{\pi(1)} \cdot X_{1}\right) \cdots\left(h_{\pi(n)} \cdot X_{n}\right)=0
$$

где $\gamma_{\pi}^{\prime}= \pm \gamma_{\pi^{-1}}$, причем теперь $h_{1}, \ldots, h_{n} \in H$ - фиксированные, а $X_{1}, \ldots, X_{n} \in V$ - переменные.

Индукцией по $m$ доказываем утверждение: если $h_{1}, \ldots, h_{m} \in H$ удовлетворяют условию

$$
F^{\prime}\left(X_{1}, \ldots, X_{m} ; h_{1}, \ldots, h_{m}\right)=\sum_{\pi \in S_{m}} \gamma_{\pi}^{\prime}\left(h_{\pi(1)} \cdot X_{1}\right) \cdots\left(h_{\pi(m)} \cdot X_{m}\right)=0
$$

для всех $X_{1}, \ldots, X_{m} \in V$, где $\gamma_{1}^{\prime} \neq 0$, то $h_{1}, \ldots, h_{m}$ линейно зависимы по модулю $\delta_{V}^{m^{2}}(H)$.

Если $m=1$, получаем $h_{1} \cdot X_{1}=0$ для всех $X_{1} \in V$, так что $h_{1} \in \delta_{V}^{0}(H)$. Пусть теперь $m>1$. Разлагая левую часть (5)

$$
F^{\prime}\left(X_{1}, \ldots, X_{m} ; h_{1}, \ldots, h_{m}\right)=\sum_{i=1}^{m}\left(h_{i} \cdot X_{1}\right) F_{i}^{\prime}\left(X_{2}, \ldots, X_{m} ; h_{1}, \ldots, \widehat{h}_{i}, \ldots, h_{m}\right)
$$

по предположению индукции получаем, что либо $h_{2}, \ldots, h_{m}$ линейно зависимы по модулю $\delta_{V}^{(m-1)^{2}}(H)$, либо мы можем найти такие $v_{2}, \ldots, v_{m} \in V$, что $F_{1}^{\prime}\left(v_{2}, \ldots, v_{m}\right.$; $\left.h_{2}, \ldots, h_{m}\right) \neq 0$.

Обозначим $f_{i}^{\prime}=F_{i}^{\prime}\left(v_{2}, \ldots, v_{m} ; h_{1}, \ldots, \widehat{h}_{i}, \ldots, h_{m}\right) \in A, i=1, \ldots, m$. Тогда $f_{1}^{\prime} \neq 0$, а из $(5)$ и $(6)$ вытекает тождество

$$
\sum_{i=1}^{m}\left(h_{i} \cdot X\right) f_{i}^{\prime}=0 \quad \forall X \in V
$$

Полагая $W=\left\langle h_{i} \cdot v_{j} \mid i=1, \ldots, m, j=2, \ldots, m\right\rangle$, завершаем доказательство, как в первой части.

Если теперь $H=\mathbb{k} G$ - групповая алгебра группы $G$, мы можем ввести дельта-множества для действия $G$ на $V$

$$
\delta_{V}^{m}(G)=\{g \in G \mid \operatorname{dim}((g-1) \cdot V) \leqslant m\}, \quad \delta_{V}(G)=\bigcup_{m} \delta_{V}^{m}(G), \quad m=0,1 \ldots
$$

Следующие свойства вытекают непосредственно из определения. 
Лемма 2.5. 1) Если $g \in \delta_{V}^{i}(G), g^{\prime} \in \delta_{V}^{j}(G)$, mo $g g^{\prime} \in \delta_{V}^{i+j}(G)$.

2) Все $\delta_{V}^{m}(G)$ содержат 1 и замкнуты относительно взятия обратного әлемента.

3) Все $\delta_{V}^{m}(G)$ инвариантны относительно внутренних автоморфизмов $G$.

В частности, $\delta_{V}(H)$ - нормальная подгруппа $G$. Напомним также определение индекса подмножества, не обязательно являющегося подгруппой.

ОПРЕДЕЛЕниЕ 2.6. Пусть $G$-группа, $T \subset G$ - подмножество. Чтобы не делать различия между левьм и правым индексами, предположим, что $T$ замкнуто относительно взятия обратного элемента. Мы будем писать $(G: T)=n$, если найдутся такие $g_{1}, \ldots, g_{n} \in G$, что $G=g_{1} T \cup \cdots \cup g_{n} T$, причем $n$ - минимальное с этим свойством.

Аналогично предложению 2.4 может быть получено следуюшее

ПРЕДЛОЖЕНИЕ 2.7. Пусть группа $G$ действует автоморфизмами ассоциативной алгебры $A$ с единицей. Пусть $G$-подмодуль $V \subset A$ не содержит 1 и порождает $A$ как алгебру с единицей. Предположим далее, что определяемая $V$ градуированная алгебра gr $A$ изоморфна $S(V)$ или $S(V) / \operatorname{ideal}\left(V^{[p]}\right)$ ( в случае char $\mathbb{k}=p>0$ ). Тогда из выполнения "слабого" тождества (1) степени п для любъх $h_{1}, \ldots, h_{n} \in \mathbb{k} G, X_{1}, \ldots, X_{n} \in A$ вытекает $\left(G: \delta_{V}^{n^{3}}(G)\right)<n$.

ДокАЗАТЕЛьСтво. Аналогично доказательству теоремы 6.1 в [3].

ЗАмЕчАниЕ 2.8. Следует отметить, что выполнения (1) только для элементов $X_{1}, \ldots, X_{n}$ из $V$ недостаточно (см. [3; пример 5.1]).

K сожалению, доказательство из [3] не проходит в случае gr $A \cong \Lambda(V)$ из-за некоммутативности. Поскольку именно этот случай нам потребуется для изучения smash-произведения универсальной обертывающей супералгебры Ли и групповой алгебры, мы дадим другое доказательство, требуюшее дополнительных предположений, но даюшее результат, достаточньй для наших целей (предложения 2.12 и 2.13$)$.

Лемма 2.9. Пусть ииклическая группа $G=\langle g\rangle$ действует автоморфизмами алгебры $\Lambda(V)$, причем $V$ является $G$-инвариантныц. Предположим, что найдутся такие линейно независимые $v_{i} \in V, i=0, \ldots, N_{1}=n^{2}-1$, что $g \cdot v_{i}=v_{i+1}, i=0, \ldots, N_{1}-1$. Тогда не может выполняться никакое нетривиальное "слабое" тождество (1) степени п для любъх $h_{1}, \ldots, h_{n} \in \mathbb{k} G$, $X_{1}, \ldots, X_{n} \in \Lambda(V)$.

ДокАЗАТЕльство. Предположим противное. Подставим в тождество (1) $h_{i}=g^{i-1}, X_{i}=v_{n(i-1)}$, тогда

$$
\begin{aligned}
\sum_{\pi \in S_{n}} \gamma_{\pi} \prod_{i=1}^{n}\left(h_{i} \cdot X_{\pi(i)}\right) & =\sum_{\pi \in S_{n}} \gamma_{\pi} \prod_{i=1}^{n} v_{n(\pi(i)-1)+i-1} \\
& =\sum_{\pi \in S_{n}}(-1)^{\operatorname{sgn} \pi} \gamma_{\pi} \prod_{i=1}^{n} v_{n(i-1)+\pi^{-1}(i)-1}=0 .
\end{aligned}
$$

В силу линейной независимости упорядоченных мономов $\prod_{i=1}^{n} v_{n(i-1)+\pi^{-1}(i)-1}$, $\pi \in S_{n}$, заключаем, что $\gamma_{\pi}=0$ для всех $\pi \in S_{n}$. Противоречие. 
ЛЕмма 2.10. Пусть ииклическая группа $G=\langle g\rangle$ действует автоморфизмами алгебры $\Lambda(V)$, причем $V$ является $G$-инвариантныц. Предположим, что $\lambda \in \mathbb{k}$ не является корнем из 1 степени меньше $n^{n}$ и $g$ имеет в $V$ не менее $N_{2}=\left(n^{n}-1\right) /(n-1)$ линейно независимых собственных векторов $c$ собственным значением $\lambda$. Тогда не может выполняться никакое нетривиальное “слабое" тождество (1) степени $n$ для любъх $h_{1}, \ldots, h_{n} \in \mathbb{k} G$, $X_{1}, \ldots, X_{n} \in \Lambda(V)$.

ДокАЗАТЕльСтво. Предположим противное и выберем такие линейно независимые $v_{i, j} \in V, i=1, \ldots, n, j=1, \ldots, s_{i}=n^{i-1}$, что $g \cdot v_{i, j}=\lambda v_{i, j}$ для всех $i, j$ (всего $N_{2}$ векторов). Зафиксируем натуральное $k$ и подставим в $(1) h_{i}=g^{k(i-1)}$ и $X_{i}=v_{i, 1} \cdots v_{i, s_{i}}$

$$
\begin{aligned}
\sum_{\pi \in S_{n}} \gamma_{\pi} \prod_{i=1}^{n}\left(h_{i} \cdot X_{\pi(i)}\right) & =\sum_{\pi \in S_{n}} \gamma_{\pi} \prod_{i=1}^{n}\left(\lambda^{k(i-1) s_{\pi(i)}} v_{\pi(i), 1} \cdots v_{\pi(i), s_{\pi(i)}}\right) \\
& =\left(\sum_{\pi \in S_{n}} \gamma_{\pi} \varepsilon(\pi) \prod_{i=1}^{n} \lambda^{k\left(\pi^{-1}(i)-1\right) s_{i}}\right) \prod_{i=1}^{n}\left(v_{i, 1} \cdots v_{i, s_{i}}\right)=0
\end{aligned}
$$

где $\varepsilon(\pi)= \pm 1$. Отсюда получаем, что полином

$$
f(t)=\sum_{\pi \in S_{n}} \gamma_{\pi} \varepsilon(\pi) t^{\sum_{i=1}^{n}\left(\pi^{-1}(i)-1\right) s_{i}}
$$

имеет $n^{n}$ различных корней $\lambda^{k}, k=1, \ldots, n^{n}$. При этом

$$
\operatorname{deg} f \leqslant \sum_{i=1}^{n}(n-1) n^{i-1}=n^{n}-1,
$$

откуда $f(t)=0$. Поскольку все числа $\sum_{i=1}^{n}\left(\pi^{-1}(i)-1\right) s_{i}, \pi \in S_{n}$, различны, получаем $\gamma_{\pi}=0$ для всех $\pi \in S_{n}$. Противоречие.

ЛЕмма 2.11. Пусть ииклическая группа $G=\langle g\rangle$ действует автоморфизмами алгебры $\Lambda(V)$, причем $V$ является $G$-инвариантныцм. Предположим, что char $\mathbb{k}=0$ и что найдутся такие линейно независимые $x_{i, j}, y_{i, j} \in V$, $i=1, \ldots, n, j=1, \ldots, i$, всего $N_{3}=n(n+1) / 2$ nар, ито $g \cdot x_{i, j}=x_{i, j}+y_{i, j}$ $u g \cdot y_{i, j}=y_{i, j}$ для всех $i, j$. Тогда не может выполняться никакое нетривиальное "слабое" тождество (1) степени п для любъх $h_{1}, \ldots, h_{n} \in \mathbb{k} G$, $X_{1}, \ldots, X_{n} \in \Lambda(V)$.

ДокАЗАТЕльство. Зафиксируем натуральные $k_{1}, \ldots, k_{n}$ и подставим $h_{i}=g^{k_{i}}$ и $X_{i}=x_{i, 1} \cdots x_{i, i}$ в (1)

$$
\begin{aligned}
& \sum_{\pi \in S_{n}} \gamma_{\pi} \prod_{i=1}^{n}\left(h_{i} \cdot X_{\pi(i)}\right) \\
& \quad=\sum_{\pi \in S_{n}} \gamma_{\pi} \prod_{i=1}^{n}\left(\left(x_{\pi(i), 1}+k_{i} y_{\pi(i), 1}\right) \cdots\left(x_{\pi(i), \pi(i)}+k_{i} y_{\pi(i), \pi(i)}\right)\right)=0 .
\end{aligned}
$$


Выбирая лишь члены, не содержашие $x_{i, j}$, получаем

$$
\begin{aligned}
& \sum_{\pi \in S_{n}} \gamma_{\pi} \prod_{i=1}^{n}\left(k_{i}^{\pi(i)} y_{\pi(i), 1} \cdots y_{\pi(i), \pi(i)}\right) \\
& \quad=\left(\sum_{\pi \in S_{n}} \gamma_{\pi} \varepsilon(\pi) \prod_{i=1}^{n} k_{i}^{\pi(i)}\right) \prod_{i=1}^{n}\left(y_{i, 1} \cdots y_{i, i}\right)=0
\end{aligned}
$$

где $\varepsilon(\pi)= \pm 1$. Отсюда получаем, что полином

$$
f\left(t_{1}, \ldots, t_{n}\right)=\sum_{\pi \in S_{n}} \gamma_{\pi} \varepsilon(\pi) t_{1}^{s_{\pi}(1)} \cdots t_{n}^{s_{\pi}(n)}
$$

обращается в 0 на всех наборах натуральных чисел $\left(k_{1}, \ldots, k_{n}\right)$. Поскольку char $\mathbb{k}=0$, то $f\left(t_{1}, \ldots, t_{n}\right)=0$, что влечет $\gamma_{\pi}=0$ для всех $\pi \in S_{n}$. Противоречие.

ПРЕДЛОЖЕНИЕ 2.12. Пусть ииклическая группа $G=\langle g\rangle$ действует автоморфизмами алгебры $\Lambda(V)$, причем $V$ является $G$-инвариантным. Предположим, что выполняется нетривиальное "слабое" тождество (1) степени $n$ для любъх $h_{1}, \ldots, h_{n} \in \mathbb{k} G, X_{1}, \ldots, X_{n} \in \Lambda(V)$. Тогда существует такое $N$, зависящее только от $n$, что $\operatorname{dim}\left(\left(g^{N}-1\right) \cdot V\right) \leqslant N$.

ДокАЗАТЕльство. Без ограничения обшности считаем $\mathbb{k}$ алгебраически замкнутым. Тогда в силу леммы 2.9 мы можем разложить $V$ в прямую сумму корневых подпространств $V(\lambda)$ оператора $g$, причем число различных собственных значений $\lambda$ не превосходит $N_{1}$. Если $\lambda$ не является корнем из 1 степени меньше $n^{n}$, то по лемме 2.10 собственное подпространство $V^{\lambda}$ имеет размерность меньше $N_{2}$, откуда снова по лемме 2.9 размерность $V(\lambda)$ меньше $N_{1} N_{2}$. Таким образом, суммарная размерность $V(\lambda)$ с $\lambda$, не являюшимся корнем из 1 степени менњше $n^{n}$, будет меньше $N_{1}^{2} N_{2}$. Отбрасывая эти корневые подпространства и заменяя $g$ на $g^{N_{4}}$, где $N_{4}=\operatorname{HOK}\left(1, \ldots, n^{n}\right)$, мы можем считать, что $g$ на $V$ имеет лишш собственное значение 1 . Снова применяя лемму 2.9 , получаем $(g-1)^{N_{1}} \cdot V=0$. Если char $\mathbb{k}=p>0$, то отсюда вытекает, что $\left(g^{p^{s}}-1\right) \cdot V=0$, где $p^{s} \geqslant N_{1}$, и предложение доказано. Так что в дальнейшем мы можем считать char $\mathbb{k}=0$. Применяя теперь лемму 2.11, мы получаем, что число линейно независимых циклических подпространств $g$ размерности больше или равной 2 не может достигать $N_{3}$. Следовательно, собственное подпространство $V^{1}$ имеет коразмерность меньше $N_{1} N_{3}$, откуда $\operatorname{dim}((g-1) \cdot V)<N_{1} N_{3}$.

ПРЕДЛОЖЕНИЕ 2.13. Пусть абелева группа $G$ действует автоморфизмами ассочиативной алгебры $A$ с единицей над полем $\mathbb{k}$ характеристики 0. Пусть G-подмодуль $V \subset A$ не содержит 1 и порождает $A$ как алгебру $c$ единицей. Предположим далее, что определяемая $V$ градуированная алгебра $\operatorname{gr} A$ изоморфна $\Lambda(V)$. Тогда из выполнения “слабого" тождества (1) степени $n$ для любых $h_{1}, \ldots, h_{n} \in \mathbb{k} G, X_{1}, \ldots, X_{n} \in A$ вытекает $\left(G: \delta_{V}(G)\right)<M$, где $M$ - число, зависящее только от $n$. 
ДокАЗАТЕЛЬСтво. Поскольку $V$ вкладьвается в $\operatorname{gr} A$ как $G$-модуль и тождество (1) выполняется для действия $G$ на gr $A$, мы можем без ограничения обшности полагать, что $A=\operatorname{gr} A=\Lambda(V)$.

Обозначим $G_{0}=\delta_{V}(G)$, по лемме 2.5 - это подгруппа. Из предложения 2.12 тогда вытекает, что факторгруппа $G / G_{0}$ имеет конечную экспоненту $N$, зависящую только от $n$. Положим $M=N^{n}$ и будем доказывать от противного, что $\left(G: G_{0}\right)<M$. Если это не так, то найдется конечно порожденная подгруппа $G_{1} \subset G$ такая, что индекс $\left(G_{1}: G_{0} \cap G_{1}\right) \geqslant M$, но при этом конечен (в силу конечности экспоненты $\left.G / G_{0}\right)$. Тогда $G_{0} \cap G_{1}$ также конечно порождена, скажем, элементами $g_{1}, \ldots, g_{s}$. Положим

$$
W=\sum_{i=1}^{s}\left(g_{i}-1\right) \cdot V .
$$

Это конечномерное подпространство, более того, оно $G$-инвариантно (в силу абелевости $G$ ). Поскольку $G_{0} \cap G_{1}$ действует тривиально на $V / W$, мы можем рассмотреть действие

$$
G_{2}=G_{1} / G_{0} \cap G_{1}
$$

на $V / W$. Если теперь некоторый элемент $g \in G_{2}$ принадлежит $\delta_{V / W}\left(G_{2}\right)$, то его прообраз $\bar{g} \in G_{1}$ будет принадлежать $\delta_{V}\left(G_{1}\right)=G_{0} \cap G_{1}$, так что $g=1$.

Заменяя $G$ на $G_{2}$ и $V$ на $V / W$, мы оказываемся в следующей ситуации: конечная абелева группа $G$ порядка больше или равного $M$ действует на пространстве $V$ так, что $\delta_{V}(G)=\{1\}$. В частности это означает, что $G$ действует на $V$ точно. При этом тождество (1) продолжает выполняться для всех $h_{1}, \ldots, h_{n} \in \mathbb{k} G$, $X_{1}, \ldots, X_{n} \in \Lambda(V)$, и мы можем считать $\gamma_{1} \neq 0$.

Считая без ограничения общности поле $\mathbb{k}$ алгебраически замкнутым, выпишем разложение

$$
V=\bigoplus_{\chi \in \widehat{G}} V_{\chi}
$$

где $\widehat{G}$ - группа характеров групшы $G$ и $V_{\chi}=\{v \in V \mid g \cdot v=\chi(g) v \forall g \in G\}$.

Возьмем произвольный $1 \neq g_{1} \in G$. В силу точности мы можем найти $0 \neq$ $v_{1} \in V_{\chi_{1}}$, причем $\chi_{1}\left(g_{1}\right)=\lambda_{1} \neq 1$. Положим $G_{1}=\operatorname{ker} \chi_{1}$. Поскольку $N-$ экспонента $G,\left(G: G_{1}\right) \leqslant N$. Так как $(G: 1) \geqslant M=N^{n}$, то $G_{1} \neq\{1\}$ и мы можем выбрать $1 \neq g_{2} \in G_{1}$. Снова в силу точности мы найдем $0 \neq v_{2} \in V_{\chi_{2}}$, причем $\chi_{2}\left(g_{2}\right)=\lambda_{2} \neq 1$, и положим $G_{2}=G_{1} \cap \operatorname{ker} \chi_{2}$. Продолжая в том же духе, мы получим набор векторов $0 \neq v_{i} \in V_{\chi_{i}}, i=1, \ldots, n$, и набор элементов $g_{1}, \ldots, g_{n} \in G$, причем $\chi_{i}\left(g_{i}\right)=\lambda_{i} \neq 1$ и $\chi_{i}\left(g_{j}\right)=1$ для всех $j>i$.

Если мы теперь подставим $h_{i}=g_{i}-1$ и $X_{i}=v_{i}$ в тождество (1), то все члены с $\pi \neq 1$ обратятся в 0, а $\pi=1$ даст $\gamma_{1}\left(\lambda_{1}-1\right) \cdots\left(\lambda_{n}-1\right) v_{1} \cdots v_{n}$. Но этот член не может равняться 0 , поскольку $\gamma_{1} \neq 0, \lambda_{i} \neq 1$ и $v_{1}, \ldots, v_{n}$ линейно независимы (так как соответствуют разным характерам $\left.\chi_{1}, \ldots, \chi_{n}\right)$. Противоречие.

Теперь мы можем получить основной результат этого параграф̆а. 
ТЕОРема 2.14. Пусть группа $G$ действует автоморфизмами ассоциативной алгебрь $A$ с единищей над полем $\mathbb{k}$. Пусть $G$-подмодуль $V \subset A$ не содержит 1 и порождает $A$ как алгебру с единицей. Через $\operatorname{gr} A$ обозначим градуированную алгебру, ассоциированную с соответствующей $V$ фильтрацией. Предположим далее, что выполняется одно из следующих условий:

1) $\operatorname{gr} A$ изоморфна симметрической алгебре $S(V)$;

2) $\operatorname{char} \mathbb{k}=p>0 u \operatorname{gr} A$ изоморфна $S(V) / \operatorname{ideal}\left(V^{[p]}\right)$, әде $V^{[p]}=\left\langle v^{p} \mid v \in V\right\rangle$;

3) char $\mathbb{k}=0, G$ абелева $u \operatorname{gr} A$ изоморфна внешней алгебре $\Lambda(V)$.

Тогда из выполнения нетривиального “слабого" тожсества вида (1) для любъх $h_{1}, \ldots, h_{n} \in \mathbb{k} G, X_{1}, \ldots, X_{n} \in A$ вытекает, что существует такая подгруппа $G_{1} \subset G$ конечного индекса и такое $G$-инвариантное подпространство $V_{1} \subset V$ конечной коразмерности, что пространство $\left(\mathbb{k} G_{1}\right)^{+} \cdot V_{1}$ конечномерно (әде, как обично, $(\mathbb{k} G)^{+}$обозначает пополняющий идеал $\left.\mathbb{k} G\right)$.

Для доказательства нам потребуются следующие факты (см. [5], [3] и [11] соответственно).

ЛЕмма 2.15. Пусть подмножество $T$ группь $G$ содержит 1 и замкнуто относительно взятия обратного элемента. Если $(G: T) \leqslant m$, то

$$
T^{4^{m}}=\underbrace{T \cdot \ldots \cdot T}_{4^{m}}
$$

является подгруппой $G$.

ЛЕмма 2.16. Пусть подмножсество $T$ векторного пространства $V$ замкнуто относительно умнохсения на скалярь. Если $\operatorname{dim} V / T \leqslant m$, то

$$
4^{m} \cdot T=\underbrace{T+\cdots+T}_{4^{m}}
$$

является подпространством в $V$.

ТЕОРема 2.17. Пусть $U, V$ и $W$ - векторные пространства над полем $\mathbb{k}$, $\varphi: U \times V \rightarrow W$ - билинейное отображение. Допустим, что существуют такие натуральные числа $m$ и $n$, что $\operatorname{dim} \varphi(u, V) \leqslant m$ для всякого $и \in U u$ $\operatorname{dim} \varphi(U, v) \leqslant n$ для всякого $v \in V$. Тогда $\operatorname{dim} \varphi(U, V) \leqslant m n$.

ДокАЗАТЕЛЬСтво теоремы 2.14. Пусть $n$ - степень “слабого" тождества. Согласно предложению 2.4 и леммам 2.16 и 2.2 множество $V_{1}=4^{n} \cdot \delta_{\mathbb{k} G}^{n^{2}}(V)$ является $G$-инвариантньм подпространством $V$ коразмерностии меньше $n$ и содержится в $\delta_{\mathbb{k} G}^{4^{n} n^{2}}(V)$. Аналогично, множество $I=4^{n} \cdot \delta_{V}^{n^{2}}(\mathbb{k} G)$ является идеалом $\mathbb{k} G$ коразмерности менњше $n$ и содержится в $\delta_{V}^{4^{n}} n^{2}(\mathbb{k} G)$. Применяя теорему 2.17 к билинейному отображению $: I \times V_{1} \rightarrow V_{1}$, получаем $\operatorname{dim}\left(I \cdot V_{1}\right) \leqslant 16^{n} n^{4}$.

Аналогично из предложения 2.7 и лемм 2.15 и 2.5 в случаях 1) и 2) вытекает, что индекс подгруппы $G_{1}=\delta_{V}(G) \subset G$ конечен. В случае 3$)$ предложение 2.13 приводит к тому же результату.

Поскольку $\operatorname{dim}\left(\mathbb{k} G_{1}\right)^{+} / I \cap\left(\mathbb{k} G_{1}\right)^{+} \leqslant \operatorname{dim} \mathbb{k} G / I<n$, мы можем выбрать такие элементы $g_{1}, \ldots, g_{n} \in G_{1}$, что

$$
\left(\mathbb{k} G_{1}\right)^{+}=\operatorname{span}\left(g_{1}-1, \ldots, g_{n}-1\right)+I \cap\left(\mathbb{k} G_{1}\right)^{+},
$$


откуда получаем

$$
\left(\mathbb{k} G_{1}\right)^{+} \cdot V_{1} \subset\left(g_{1}-1\right) \cdot V_{1}+\cdots+\left(g_{n}-1\right) \cdot V_{1}+I \cdot V_{1}
$$

По построению пространство в правой части конечномерно.

ЗАмЕЧАнИЕ 2.18. Из леммы 2.2 также вытекает, что построенное нами подпространство $V_{1}$ инвариантно относительно любого гомоморфизма $G$-модулей $\Phi: V \rightarrow V$.

\section{§3. Доказательство основной теоремы}

Докажем сначала достаточность условий 1)-4) теоремы 1.5 для наличия тождества в $R=U(L) \# \mathbb{k} G$. Прежде всего заметим, что $L_{0} \oplus M$ является $G$-инвариантной подсупералгеброй $L$ (даже идеалом), и рассмотрим подкольцо

$$
R_{1}=U\left(L_{0} \oplus M\right) \# \mathbb{k} A
$$

кольца $R$. Из теоремы Пуанкаре-Биркгофа-Витта для супералгебр и определения smash-произведения вытекает, что $R$ порождается как левый $R_{1}$-модуль элементами вида

$$
x_{i_{1}} \cdots x_{i_{s}} \# g_{j}, \quad i_{1}<\cdots<i_{s}, \quad s=0,1, \ldots,
$$

где $\left\{x_{i}\right\}$ - базис $L_{1}$ по модулю подпространства $M$, a $\left\{g_{j}\right\}$ - представители правых смежных классов $G$ по подгруппе $A$. Поскольку число элементов вида $(8)$ конечно, нам достаточно доказать, что $R_{1}$ удовлетворяет тождеству.

Рассмотрим идеал $I \triangleleft R_{1}$, порожденньй $N$. Поскольку $N$ является $A$-инвариантным идеалом супералгебры Ли $L_{0} \oplus M$, мы видим, что

$$
I=R_{1} N=N R_{1}
$$

В силу теоремы Пуанкаре-Биркгофа-Витта $N^{\operatorname{dim} N+1}=0$, откуда $I^{\operatorname{dim} N+1}=0$, так что $I$ - нильпотентньй идеал. Далее,

$$
R_{1} / I \cong U\left(L_{0} \oplus M / N\right) \# \mathbb{k} A \cong U\left(L_{0}\right) \otimes \Lambda(M / N) \otimes \mathbb{k} A
$$

поскольку действие $A$ и $L_{0}$ на $M / N$ тривиально. Наконец, $U\left(L_{0}\right)$ и $\mathbb{k} A$ коммутативны, так что $R_{1} / I$ удовлетворяет тождеству $[[X, Y], Z]=0$. Поскольку идеал $I$ нильпотентен, $R_{1}$ также является $P I$-алгеброй, что и требовалось.

Переходим к доказательству необходимости. Из наличия тождества в $U(L) \# \mathbb{k} G$ (а следовательно, и в $U(L)$ и $\mathbb{k} G$ ) нам известна структура $L$ и $G$ (см. теоремы 1.2 и 1.3). А именно, имеется абелева подгруппа $A_{1} \subset G$ конечного индекса, $L_{0}$ является абелевой, а также найдется $L_{0}$-подмодуль $M \subset L_{1}$, удовлетворяющий следуюшим условиям:

$$
[M, M]=0, \quad L_{1} / M \text { и }\left[L_{0}, M\right] \text { конечномерны. }
$$


Для нашей цели нам необходимо выбрать подпространство $M \subset L_{1}$, удовлетворяющее условиям (9), не только $L_{0^{-}}$, но и $G$-инвариантньм. Напомним основные этапы доказательства теоремы 1.2 в [4], чтобы убедиться, что на каждом шаге получается характеристическое (т.е. устойчивое относительно всех автоморфизмов супералгебры $L)$ подпространство в $L_{1}$.

Прежде всего введем три типа дельта-множеств (четвертая возможность не представляет интереса в силу абелевости $\left.L_{0}\right)$

$$
\begin{array}{ll}
\delta_{L_{1}}^{m}\left(L_{0}\right)=\left\{x \in L_{0} \mid \operatorname{dim}\left[x, L_{1}\right] \leqslant m\right\}, & \delta_{L_{1}}\left(L_{0}\right)=\bigcup_{m} \delta_{L_{1}}^{m}\left(L_{0}\right), \\
\delta_{L_{0}}^{m}\left(L_{1}\right)=\left\{x \in L_{1} \mid \operatorname{dim}\left[L_{0}, x\right] \leqslant m\right\}, & \delta_{L_{0}}\left(L_{1}\right)=\bigcup_{m} \delta_{L_{0}}^{m}\left(L_{1}\right), \\
\delta_{L_{1}}^{m}\left(L_{1}\right)=\left\{x \in L_{1} \mid \operatorname{dim}\left[L_{1}, x\right] \leqslant m\right\}, & \delta_{L_{1}}\left(L_{1}\right)=\bigcup_{m} \delta_{L_{1}}^{m}\left(L_{1}\right),
\end{array}
$$

где $m=0,1, \ldots$.

В [4] доказывается, а такженепосредственно видно из существования какого-нибудь подпространства $M \subset L_{1}$, удовлетворяющего условиям (9), что существуют такие $s, t_{0}$ и $t_{1}$, что

$$
\begin{aligned}
& \delta_{L_{1}}\left(L_{0}\right)=\delta_{L_{1}}^{s}\left(L_{0}\right)=L_{0}, \\
& \delta_{L_{0}}\left(L_{1}\right)=\delta_{L_{0}}^{t_{0}}\left(L_{1}\right) \text { имеет конечную коразмерность в } L_{1}, \\
& \delta_{L_{1}}\left(L_{1}\right)=\delta_{L_{1}}^{t_{1}}\left(L_{1}\right) \text { имеет конечную коразмерность в } L_{1},
\end{aligned}
$$

причем по определению все эти подпространства Aut $L$-инвариантны.

Положим $Q=\delta_{L_{0}}\left(L_{1}\right) \cap \delta_{L_{1}}\left(L_{1}\right) \subset L_{1}$. Это Aut $L$-инвариантное подпространство конечной коразмерности. Из тождества Якоби вытекает, что оно также $L_{0}$-инвариантно. По теореме 2.17 получаем

$$
\operatorname{dim}\left[L_{0}, Q\right] \leqslant s t_{0}, \quad \operatorname{dim}[Q, Q] \leqslant t_{1}^{2} .
$$

Далее, в силу (10) централизатор $C_{L_{1}}(x)$ любого элемента $x \in L_{0}$ имеет конечную коразмерность в $L_{1}$, так что $C_{L_{1}}([Q, Q])$ также имеет конечную коразмерность. Поскольку $Q$ является Aut $L$ - и $L_{0}$-инвариантным, этими же свойствами обладает $C_{L_{1}}([Q, Q])$. Обозначим $T=Q \cap C_{L_{1}}([Q, Q])$, тогда $T$ - подпространство $L_{1}$ конечной коразмерности, устойчивое относительно Aut $L$ и $L_{0}$.

Наконец, положим $\widetilde{L}=[T, T] \oplus T$ и $M=Z(\widetilde{L}) \cap T$. Тогда из построения следует, что $\widetilde{L}$ - подсупералгебра $L$, причем компонента $\widetilde{L}_{0}=[T, T]$ конечномерна и центральна в $\widetilde{L}$. Из леммы 5.2 в [4] и следуюшего за ней рассуждения теперь вытекает, что $Z(\widetilde{L})$ имеет конечную коразмерность в $\widetilde{L}$ (здесь также используется наличие тождества в $U(\widetilde{L}) \subset U(L))$. Следовательно, наше подпространство $M$ имеет конечную коразмерность в $T$, а стало быть, и в $L_{1}$. По построению $[M, M]=0$, $\left[L_{0}, M\right] \subset\left[L_{0}, Q\right]$ конечномерно и, как мы и хотели, $M$ является Aut $L$ - и $L_{0}$-инвариантным.

Возврашаясь к рассмотрению smash-произведения $U(L) \# \mathbb{k} G$, мы видим, что $L_{0} \oplus M$ является $G$-инвариантной подсупералгеброй $L$, причем $L_{1} / M$ конечномерно. Следовательно, мы можем без ограничения обшности считать, что $M=L_{1}$, 
так что $\left[L_{1}, L_{1}\right]=0$ и пространство $\left[L_{0}, L_{1}\right]$ конечномерно. Из теоремы 1.4 мы знаем, что абелева подгруппа $A_{1} \subset G$ конечного индекса может быть выбрана действующей тривиально на $L_{0}$. Более того, из $\left[L_{1}, L_{1}\right]=0$ вытекает, что алгебра $\Lambda\left(L_{1}\right) \# \mathbb{k} A_{1}$ вложена в $U(L) \# \mathbb{k} G$, и из наличия тождества в последней получаем, что выполняется некоторое тождество на действие $A_{1}$ на $\Lambda\left(L_{1}\right)$. Согласно теореме 2.14 мы можем найти такую подгруппу $A_{2} \subset A_{1}$ конечного индекса и такое $A_{1}$-инвариантное подпространство $V_{1} \subset L_{1}$ конечной коразмерности, что $\left(\mathbb{k} A_{2}\right)^{+} \cdot V_{1}$ конечномерно. Поскольку $A_{1}$ действует тривиально на $L_{0}$, операторы из $\operatorname{ad} L_{0}$ перестановочны с действием $A_{1}$ на $L_{1}$, так что согласно замечанию 2.18 мы можем считать $V_{1}$ ad $L_{0}$-инвариантньп. Далее, положим

$$
A=\bigcap_{g \in G} g A_{2} g^{-1},
$$

тогда по построению $A$ - нормальная абелева подгруппа в $G$ конечного индекса, действуюшая тривиально на $L_{0}$, причем $(\mathbb{k} A)^{+} \cdot V_{1}$ конечномерно для выбранного нами $V_{1}$.

Применим теперь “процесс усреднения” к $V_{1}$, полагая

$$
M=\sum_{g \in G} g \cdot V_{1}
$$

Тогда $M-G$ - и $L_{0}$-инвариантное подпространство $L_{1}$ конечной коразмерности, причем вьшшеуказанная сумма имеет на самом деле лишш конечное число различных слагаемых, поскольку подгруппа $A$ конечного индекса оставляет $V_{1}$ инвариантным. Следовательно, подпространство $(\mathbb{k} A)^{+} \cdot M \subset M$ все еще конечномерно и, более того, в силу нормальности $A$ в $G$ оно является $G$-инвариантньм. Наконец, из перестановочности операторов из $\operatorname{ad} L_{0}$ с действием $A$ вытекает, что $(\mathbb{k} A)^{+} \cdot M$ будет и $L_{0}$-инвариантным. Остается положить

$$
N=\left[L_{0}, M\right]+(\mathbb{k} A)^{+} \cdot M
$$

Тогда подпространство $N \subset M$ конечномерно, $G$ - и $L_{0}$-инвариантно и по построению $A$ и $L_{0}$ действуют тривиально на $M / N$, что и завершает доказательство теоремы.

В заключение автор выражает благодарность Ю. А. Бахтурину за постановку задачи и внимание к работе.

\section{Список литературы}

1. Montgomery S. Hopf algebras and their actions on rings. Providence, RI: Amer. Math. Soc., 1993. (CBMS Reg. Conf. Ser. Math. V. 82.)

2. Passman D.S. Infinite crossed products. Boston: Academic Press, 1989. (Pure Appl. Math. V. 135.)

3. Bahturin Yu., Petrogradsky V. Polynomialidentities in smash products // J. Lie Theory. 2002. V. 12. № 2. P. 369-395.

4. Бахтурин Ю. А. Тождества универсальной обертьвающей алгебры для супералгебры Ли // Матем. сб. 1985. Т. 127 (169). № 3 (7). С. 384-397.

5. Passman D. Group rings satisfying a polynomial identity // J. Algebra. 1972. V. 20. P. 103-117. 
6. Петроградский B. М. О существовании тождества в ограниченной обертывающей // Матем. заметки. 1991. Т. 49. № 1. С. 84-93.

7. Petrogradski V. M. Identities in the enveloping algebras for modular Lie superalgebras // J. Algebra. 1992. V. 145. № 1. P. 1-21.

8. Kochetov $M$. On identities for coalgebras and Hopf algebras // Comm. Algebra. 2000. V. 28. № 3. P. 1211-1221.

9. Handelman D., Lawrence J., Schelter W. Skew group rings // Houston J. Math. 1978. V. 4. № 2. P. 175-197.

10. Kochetov M. PI Hopf algebras of prime characteristic // J. Algebra (to appear).

11. Neumann P. An improved bound for BFC groups // J. Austral. Math. Soc. 1970. V. 11. P. 19-27.

Московский государственнњй университет им. М.В. Ломоносова

E-mail: mkotchetov@hotmail.com
Поступила в редакцию 25.04 .2002 